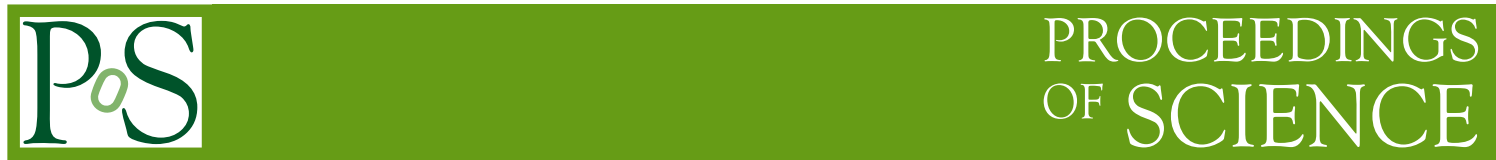

\title{
Chiral random matrix theory for staggered fermions
}

\section{James C. Osborn*}

Argonne Leadership Computing Facility, Argonne National Laboratory, Argonne, IL 60439, USA

E-mail: osbornealcf.anl.gov

We present a completed random matrix theory for staggered fermions which incorporates all taste symmetry breaking terms at their leading order from the staggered chiral Lagrangian. This is an extension of previous work which only included some of the taste breaking terms. We will also discuss the effects of taste symmetry breaking on the eigenvalues in the weak and strong taste breaking limits, and compare with some results from lattice simulations.

The XXIX International Symposium on Lattice Field Theory

July 10-16, 2011

Squaw Valley, Lake Tahoe, California

\footnotetext{
* Speaker.
} 


\section{Introduction}

Staggered fermions are an inexpensive and popular way of discretizing quarks on a space-time lattice. They preserve a chiral symmetry with a local action at the expense of containing extra modes that quadruple the number of quark species being simulated. The quadrupled species, called tastes, are mixed at nonzero lattice spacing, but are expected to produce four independent quark flavors in the continuum limit. Evidence of this behavior can be seen in the low eigenvalues of the staggered Dirac matrix. At small lattice spacings the eigenvalues tend to cluster into distinct quartets which represent the four tastes. As the lattice spacing is decreased, the eigenvalues within a quartet will become more degenerate [1].

In [2] a Random Matrix Theory (RMT) for staggered lattice fermions was introduced to describe the low eigenvalues of the staggered Dirac operator. The staggered RMT (SRMT) adds additional terms to the standard chiral Random Matrix Theory that have the appropriate symmetries of the lattice operator. These additional terms in the SRMT reproduce the known $O\left(a^{2}\right)$ (ignoring any extra factors of $\alpha_{s}$ which we drop for convenience) taste breaking terms that appear in the staggered chiral Lagrangian.

The SRMT is equivalent to the staggered chiral Lagrangian at zero momentum. This equivalence has been demonstrated for the fermionic partition function, but is only conjectured for the partially quenched case, which is related to the eigenvalues of the Dirac operator. Here we will directly compare estimates of the size of the taste breaking in lattice simulations determined from the staggered chiral Lagrangian (in the $p$-regime) with estimates obtained from comparing low eigenvalues to the SRMT predictions (in the $\varepsilon$-regime). Our initial tests show good agreement with the predictions of SRMT in support of the conjectured equivalence of the partially quenched theories.

\section{Staggered Chiral Lagrangian}

The effective chiral Lagrangian for staggered fermions at order $a^{2}$ is given by [3, 身]

$$
\mathscr{L}=\frac{F^{2}}{8}\left\langle\partial_{\mu} U \partial_{\mu} U^{\dagger}\right\rangle-\frac{1}{2} \Sigma_{0} m\left\langle U+U^{\dagger}\right\rangle+a^{2} \mathscr{V}
$$

where $\langle X\rangle$ stands for the trace of $X$, and $F$ and $\Sigma_{0}$ are the low energy constants (LECs) related to the pion decay constant (with the convention that the physical value for $F \approx 131 \mathrm{MeV}$ ) and the magnitude of the chiral condensate, respectively. The taste breaking terms can be divided into two parts $\mathscr{V}=\mathscr{V}_{1 t}+\mathscr{V}_{2 t}$. The first part contains the single-trace terms (with $\xi_{\mu}=\gamma_{\mu}^{*}$ )

$$
\begin{aligned}
-\mathscr{V}_{1 t} & =C_{1}\left\langle\xi_{5} U \xi_{5} U^{\dagger}\right\rangle+C_{3} \frac{1}{2} \sum_{\mu}\left[\left\langle\xi_{\mu} U \xi_{\mu} U\right\rangle+\text { h.c. }\right] \\
& +C_{4} \frac{1}{2} \sum_{\mu}\left[\left\langle\xi_{\mu 5} U \xi_{5 \mu} U\right\rangle+\text { h.c. }\right]+C_{6} \sum_{\mu<v}\left\langle\xi_{\mu v} U \xi_{v \mu} U^{\dagger}\right\rangle
\end{aligned}
$$

and the second part has the two-trace terms

$$
\begin{aligned}
-\mathscr{V}_{2 t} & =C_{2 V} \frac{1}{4} \sum_{\mu}\left[\left\langle\xi_{\mu} U\right\rangle\left\langle\xi_{\mu} U\right\rangle+\text { h.c. }\right]+C_{2 A} \frac{1}{4} \sum_{\mu}\left[\left\langle\xi_{\mu 5} U\right\rangle\left\langle\xi_{5 \mu} U\right\rangle+\text { h.c. }\right] \\
& +C_{5 V} \frac{1}{2} \sum_{\mu}\left[\left\langle\xi_{\mu} U\right\rangle\left\langle\xi_{\mu} U^{\dagger}\right\rangle\right]+C_{5 A} \frac{1}{2} \sum_{\mu}\left[\left\langle\xi_{\mu 5} U\right\rangle\left\langle\xi_{5 \mu} U^{\dagger}\right\rangle\right] .
\end{aligned}
$$




\section{Staggered Chiral Random Matrix Theory}

The (fermionic) staggered chiral random matrix theory partition function can be written as [2]

$$
Z_{S R M T}=\int d W p_{0}(W) p_{T}(T) \prod_{f=1}^{N_{f}} \operatorname{det}\left(D+m_{f}\right)
$$

with

$$
D=\left(\begin{array}{cc}
0 & i W \\
i W^{\dagger} & 0
\end{array}\right) \otimes \mathbb{I}_{4}+a T
$$

where $W$ is a $(N+v) \times N$ complex matrix with $v$ the absolute value of the topological charge and $T$ incorporates the taste breaking terms. The matrix potential for $W$ is conveniently a Gaussian,

$$
p_{0}(W)=\exp \left(-\alpha N\left\langle W^{\dagger} W\right\rangle\right)
$$

with $\sqrt{\alpha}=\Sigma_{0} V / 2 N(V$ is the four volume).

The taste breaking contribution to the SRMT $(T)$ has eight terms that correspond directly with the eight taste breaking terms of the chiral Lagrangian. Its complete form was given in [2]. As an example, the $C_{4}$ term corresponds to

$$
T_{4}=\sum_{\mu}\left(\begin{array}{cc}
A_{\mu} & 0 \\
0 & B_{\mu}
\end{array}\right) \otimes \xi_{\mu 5}
$$

where $A_{\mu}$ and $B_{\mu}$ are Hermitian matrices of size $(N+v) \times(N+v)$ and $N \times N$, respectively. For convenience we can choose a Gaussian weight function for these matrices,

$$
p_{T_{4}}=\exp \left(-\frac{\alpha N^{2}}{2 V C_{4}} \sum_{\mu}\left\langle A_{\mu}^{2}\right\rangle+\left\langle B_{\mu}^{2}\right\rangle\right) .
$$

One can then show that the (fermionic) RMT with this extra matrix term is equivalent to the zeromomentum staggered chiral Lagrangian with just a $C_{4}$ taste breaking term.

Note that while the correction to the RMT enters at order $a$, this still reproduces a term of order $a^{2}$ in the chiral Lagrangian. This is due to the taste breaking terms in the SRMT being traceless. As demonstrated in [2], when expanding the determinant of the SRMT Dirac matrix, the $O(a)$ term vanishes for this reason, and results in a partition function that has a leading corrections at $O\left(a^{2}\right)$ even though the SRMT Dirac operator has terms of $O(a)$.

The two-trace terms can be incorporated in the SRMT in a couple of ways. One is to add terms such as

$$
\left(\begin{array}{cc}
b_{\mu} \mathbb{I}_{N+v} & 0 \\
0 & \pm b_{\mu} \mathbb{I}_{N}
\end{array}\right) \otimes \xi_{\mu 5}
$$

with a Gaussian weight for the scalar $b_{\mu}$. This will give a contribution to the $C_{2 A}$ and $C_{5 A}$ terms. While this term will reproduce the correct term in the chiral Lagrangian, it has no analogue in the 
lattice Dirac matrix. This term is of the form of a fluctuating taste-dependent mass, which is not present on the lattice.

An alternative form for the two-trace terms is to simply modify the potentials for the matrix terms corresponding to the one-trace terms. By replacing the simple Gaussian weight with one that also includes $\left\langle A_{\mu}\right\rangle^{2},\left\langle B_{\mu}\right\rangle^{2}$ and $\left\langle A_{\mu}\right\rangle\left\langle B_{\mu}\right\rangle$ in the exponential, the coefficients can be tuned to give the correct two-trace terms in the chiral Lagrangian [2].

\section{Generalized Staggered Random Matrix Theory}

If we look at the final form of the SRMT Dirac matrix, we see that it is the most general matrix that is consistent with the symmetries of the staggered Dirac operator; it is anti-Hermitian and anticommutes with the staggered chiral symmetry matrix $\gamma_{5} \otimes \xi_{5}$. As mentioned in the previous section, the single-trace terms from the chiral Lagrangian are reproduced by considering independent Gaussian weights for the remaining matrix elements. Meanwhile the two-trace terms can be reproduced by adding two-trace terms to the RMT weights.

In this manner one could consider generalizing the SRMT to include more terms in the weight function in an attempt to reproduce higher order terms in the chiral Lagrangian (at zero momentum). One can then draw an analogy between the formulation of a RMT and of an effective Lagrangian. For the Lagrangian one includes all terms, up to some order, that are consistent with the symmetries. Likewise for the RMT one can consider a matrix containing all elements consistent with the symmetries of the Dirac operator, with a generalized weight function for these matrix elements up to some level of complexity. Of course the mapping from the RMT potential to the chiral Lagrangian at higher order may not be as simple as in the SRMT presented here, but one could speculate that a generalized RMT could reproduce all higher order terms of the chiral Lagrangian. Again this equivalence only holds for the zero momentum Lagrangian, but it is also possible to reduce the full Lagrangian to an effective zero momentum Lagrangian (for a recent example see [5]), which then might be representable as a RMT.

\section{Dominant form of taste breaking}

Most of the taste breaking coefficients can be measured in lattice simulations by comparing to staggered chiral perturbation theory $(\mathrm{S} \chi \mathrm{PT})$ results. The four single-trace parameters are uniquely determined from the splittings of the pion masses at leading order [ 4 . Typically the $C_{4}$ term is found to be the dominant contribution to the pion spectrum [3]. The two-trace terms enter in $\mathrm{S} \chi \mathrm{PT}$ formulas in the combinations $C_{V, A}^{ \pm}=\left(C_{2\{V, A\}} \pm C_{5\{V, A\}}\right) / 2$. They don't contribute to the pion splittings at leading order, but the $C_{A}^{-}$and $C_{V}^{-}$terms do appear in one-loop expressions, while the $C_{A}^{+}$and $C_{V}^{+}$terms do not [ $₫$. In lattice simulations $C_{A}^{-}$has been found to be larger than $C_{V}^{-}$[6].

Both of the dominant terms, $C_{4}$ and $C_{A}^{-}$, come from same term in the SRMT with the more general form for the weight function. This supports the idea of constructing the SRMT from a single matrix with the correct symmetries and with a generalized weight function. Based on the lattice measurements, the leading contribution to taste breaking in the staggered Dirac matrix has an axial-vector taste structure. If one imagined rotating the staggered Dirac matrix into a taste basis and then expanding in powers of $a$, the dominant correction at order $a$ would then have the same 


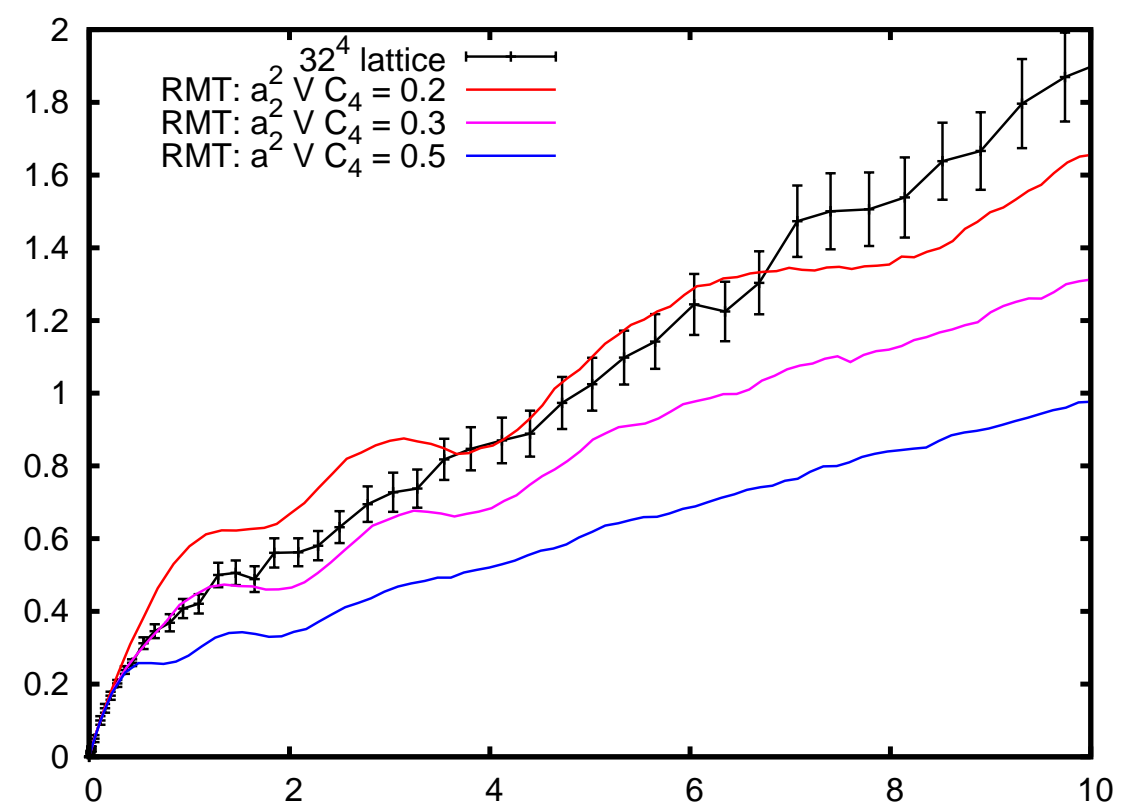

Figure 1: Number variance of the lowest eigenvalues of the $32^{4}$ lattice ensemble and the SRMT.

form as in 3.4. . Of course the exact potential for the lattice Dirac matrix would be much more complicated than in the SRMT, but the leading effects at low energy can be captured by the SRMT weight function considered here. Among the terms in the SRMT potential corresponding to the axial-vector matrix, we have no reason to favor one over the other, so we would naively expect them to be of similar magnitude. We would then expect the corresponding terms in the chiral Lagrangian to be of similar order, and also dominant over the corresponding terms with other taste symmetries, which is consistent with lattice measurements.

\section{Extracting LECs from RMT}

The RMT predictions for the eigenvalue correlations can be used to extract LECs from lattice simulations. For example $\Sigma_{0}$ can be obtained by fitting to the eigenvalue density. Additionally $F$ can be obtained from the correlations of eigenvalues with an imaginary chemical potential [7].

If the taste breaking is small enough then these methods can apply directly to staggered eigenvalues by replacing each quartet of eigenvalues with its average [1]. In this case one could also try to extract the taste breaking parameters from the splittings of the eigenvalues within the quartet. In practice, it would likely be too difficult to extract all the parameters, but if one assumes that the $C_{4}$ term is dominant, then it should be possible to estimate it from comparison to the SRMT.

However, if the taste breaking is large, then the higher order taste breaking terms can become important. In this case the effective chiral Lagrangian reduces to a single flavor for the remaining staggered chiral symmetry with a new set of LECs that are in principle unrelated to the original ones [2]. Thus extracting the LECs from low eigenvalues when there aren't clear quartets present may not yield the continuum LECs in chiral Lagrangian. 

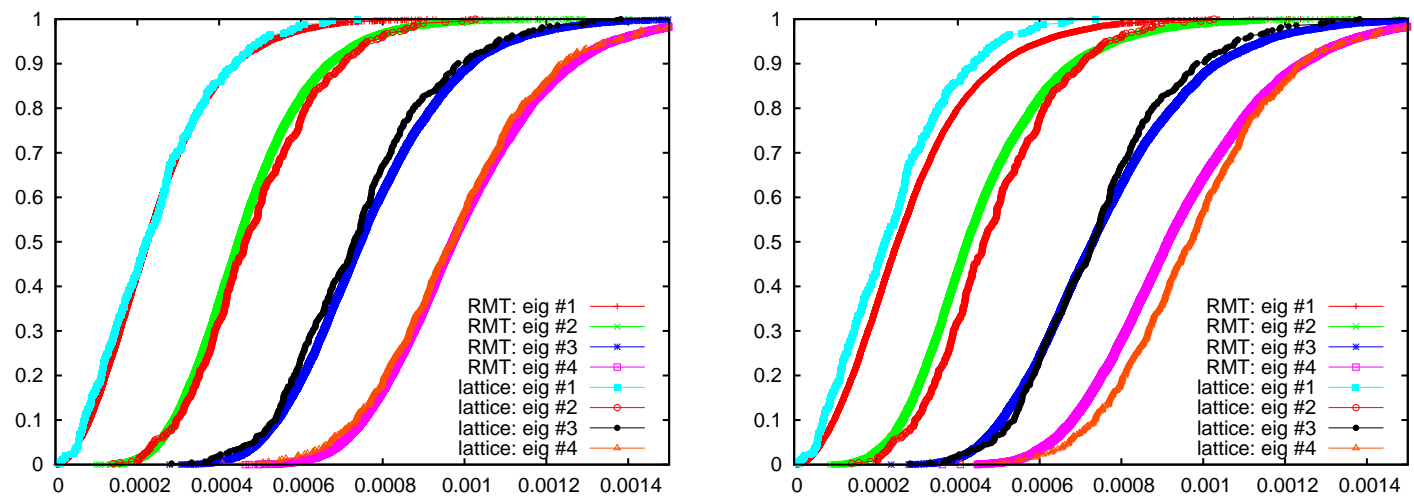

Figure 2: Individual integrated densities of the lowest four eigenvalues from the $32^{4}$ lattice ensemble and the SRMT. The dominant taste breaking parameter in the SRMT is set to $a^{2} V C_{4}=0.3$ (left) and 0.2 (right).

\section{Comparison to lattice simulations}

As an initial test of the SRMT, we will compare the leading order taste breaking term obtained from fitting the Dirac operator eigenvalues to the SRMT with that obtained from fitting the pion spectrum to the staggered chiral Lagrangian. Since it is difficult to get a single lattice ensemble that we could use for both measurements we will use two ensembles with all parameters identical except for the volume.

For the pion masses we use an ensemble from the MILC collaboration $2+1+1$ flavor HISQ runs [8]. The ensemble has a volume of $32^{3} \times 96$ with a lattice spacing of $a \approx 0.09 \mathrm{fm}$ and with a light quark mass $m_{l}=m_{s} / 5$. From the pion mass splittings we can get the single-trace taste breaking terms in the chiral Lagrangian. Using a value of $F=131 \mathrm{MeV}$, this gives

$$
a^{2} V C_{1}=0.03(8), a^{2} V C_{3}=-0.03(4), a^{2} V C_{4}=0.84(4), a^{2} V C_{6}=0.03(3) .
$$

We can see here that $C_{4}$ is clearly dominant, as expected, and that the other coefficients are all consistent with zero.

We generated a new ensemble of 430 lattices of size $32^{4}$ with all other parameters the same as the previous ensemble. From the volume scaling we expect to find

$$
a^{2} V C_{4}=0.28(1)
$$

on this new ensemble. We then compare lattice eigenvalues with numerical simulations of the SRMT with only the $C_{4}$ taste breaking term. For this comparison we choose to use the number variance statistic. This shows the fluctuations (variance) in the number of eigenvalues in an interval starting at zero versus the average number in the interval. We evaluated it numerically from simulations of the SRMT with $N=400$.

In Figure 1 we plot the number variance of the lattice eigenvalues against the SRMT at different values of $a^{2} V C_{4}$. We can see that the best agreement for small intervals (up to around 2 to 4 eigenvalues) is at $a^{2} V C_{4} \approx 0.3$. This is in good agreement with our prediction (7.2) obtained from the pion mass splittings. As the length of the interval grows, the lattice results start to move away from the SRMT result. This is likely due to higher momentum modes entering on the lattice, that aren't captured in the RMT. This happens at the QCD equivalent of the Thouless energy [9], which for this ensemble, in units of the average eigenvalue spacing, is $F^{2} \sqrt{V} \approx 3.5$. This is consistent with our observations from the number variance. 
As a further check that the SRMT describes the low eigenvalues of the staggered Dirac operator, we look at the individual integrated densities of the lowest four eigenvalues. In Figure 6 we see the integrated density from the $32^{4}$ lattice along with that from the numerical simulations of the SRMT at two different values of the taste breaking parameter $a^{2} V C_{4}=0.2,0.3$. We can see that the value of 0.3 fits the lattice data much better than at 0.2 , again confirming that the predictions of the SRMT fit the lattice data well and give estimates of the taste breaking parameters that are consistent with the staggered chiral Lagrangian.

\section{Summary}

We have shown a chiral RMT that incorporates all leading order taste breaking terms from staggered chiral Lagrangian. The SRMT can be constructed by considering a RMT with same symmetries as the staggered Dirac operator and a generalized weight function. Initial tests show that the predictions of the SRMT are in good agreement with lattice simulations within the range of validity of the SRMT. Additionally, using the predictions of the SRMT, the dominant taste breaking parameter can be extracted from the low eigenvalues of the staggered Dirac operator and gives consistent results with that obtained from the pion mass splittings.

\section{Acknowledgments}

We thank Doug Toussaint for providing the pion splitting data for the MILC ensemble. This research used resources of the Argonne Leadership Computing Facility at Argonne National Laboratory, and was supported by the U.S. DOE under contract DE-AC02-06CH11357.

\section{References}

[1] E. Follana et al., Phys. Rev. Lett. 93 (2004) 241601 [hep-lat/0406010]; S. Durr, C. Hoelbling and U. Wenger, Phys. Rev. D 70 (2004) 094502 [hep-lat/0406027]; E. Follana et al., Phys. Rev. D 72 (2005) 054501 [hep-lat/0507011].

[2] J. C. Osborn, Nucl. Phys. Proc. Suppl. 129 (2004) 886 [hep-lat/0309123]; Phys. Rev. D 83 (2011) 034505 [arXiv:1012.4837 [hep-lat]].

[3] W. -J. Lee and S. R. Sharpe, Phys. Rev. D 60 (1999) 114503 [hep-lat/9905023].

[4] C. Aubin and C. Bernard, Phys. Rev. D 68 (2003) 034014 [hep-lat/0304014].

[5] C. Lehner, S. Hashimoto and T. Wettig, JHEP 1006 (2010) 028 [arXiv:1004.5584 [hep-lat]].

[6] C. Aubin et al. [MILC Collaboration], Phys. Rev. D 70 (2004) 114501 [hep-lat/0407028].

[7] P. H. Damgaard, U. M. Heller, K. Splittorff and B. Svetitsky, Phys. Rev. D 72 (2005) 091501 [hep-lat/0508029]; G. Akemann, P. H. Damgaard, J. C. Osborn and K. Splittorff, Nucl. Phys. B 766 (2007) 34 [Erratum-ibid. B 800 (2008) 406] [hep-th/0609059].

[8] A. Bazavov, C. Bernard, C. DeTar, W. Freeman, S. Gottlieb, U. M. Heller, J. E. Hetrick and J. Laiho et al., PoS LATTICE 2010 (2010) 320 [arXiv:1012.1265 [hep-lat]].

[9] J. C. Osborn and J. J. M. Verbaarschot, Nucl. Phys. B 525 (1998) 738 [hep-ph/9803419]; J. C. Osborn and J. J. M. Verbaarschot, Phys. Rev. Lett. 81 (1998) 268 [hep-ph/9807490]. 